\title{
Viability of the $R+e^{T}$ cosmology
}

\author{
P.H.R.S. Moraes ${ }^{\dagger}$, P.K. Sahoo ${ }^{\ddagger}$, S.K.J. Pacif* \\ ${ }^{\dagger}$ Universidade de São Paulo, Instituto de Astronomia, \\ Geofísica e Ciências Atmosféricas, R. do Matão 1226, \\ Cidade Universitária, 05508-090 São Paulo, SP, Brazil * \\ ${ }^{\ddagger}$ Department of Mathematics, Birla Institute of Technology and Science-Pilani, \\ Hyderabad Campus, Hyderabad-500078, India ${ }^{\dagger}$ and \\ *Department of Mathematics, School of Advanved Sciences, \\ VIT University, Vellore 632014, Tamil Nadu, India ${ }^{\ddagger}$
}

\begin{abstract}
We propose a new theoretical approach for a cosmological model, which starts from an exponential of the trace of the energy-momentum tensor-dependence on the gravitational action, to be summed to the Ricci scalar. We derive the referred field equations and Friedmann-like equations. We derive the scale factor, Hubble parameter and deceleration parameter, in terms of both time and redshift. In possession of those parameters in terms of the redshift, we confront their predictions with the observational Hubble dataset and the outcomes are pretty satisfactory so that the model can be seen as a new alternative to the cosmological constant problem. We also present the statefinder diagnostic and discuss profoundly the dynamical behaviour of the model.

PACS numbers: 04.50.kd.
\end{abstract}

\section{INTRODUCTION}

The greatest mystery of Physics nowadays is the cause of the acceleration of the expansion of the universe. The expansion of the universe, discovered by E. Hubble in 1929 [1], was expected to be slowing down due to the attractive feature of the force of gravity. However, at the end of the 20th century, the measuring of the brightness of distant supernovae Ia [2, 3] has shown that the expansion is, in fact, speeding up. Approximately 20 years later, what causes this acceleration still remains unknown.

While the standard cosmological model, named $\Lambda \mathrm{CDM}$ model, for which $\Lambda$ represents the cosmological constant and CDM stands for cold dark matter, indeed provides a great fit with observations 4], there is a strong shortcoming in what concerns the physical interpretation of the cosmological constant [5]-[7]. Neither have dark matter particles been detected [8, 9].

Since $\Lambda$ CDM model is based on Einstein's General Theory of Relativity [10, theoretical physicists have been trying to solve the above issues by extending General Relativity. The idea is that some new degrees of freedom of extended theories of gravity could play the role of the dark sector. In fact, some extra terms in the field equations of extended gravity theories [11, 12] indeed can describe the dark energy and matter effects simply as correction terms to General Relativity.

The dark energy and matter dynamical effects have been obtained within extended gravity theories as one can check References [13, 14, and [15, 16, respectively. Naturally, those theories may also have some shortcomings. For instance, solar system tests have ruled out most of the $f(R)$ models proposed so far [17, 18, for which $f(R)$ means a general function of the Ricci scalar, to replace $R$ in the usual Einstein-Hilbert action.

\footnotetext{
*Email: moraes.phrs@gmail.com

†Email: pksahoo@hyderabad.bits-pilani.ac.in

‡Email: shibesh.math@gmail.com
}

Our proposal herewith in the present paper is to address the cosmic acceleration in an extended gravitational theory that allows the generalization of the material sector of the field equations of General Relativity, rather than their geometrical sector.

We are going to replace $R$ in the Einstein-Hilbert gravitational action by $R+f(T)$, with $T$ representing the trace of the energy-momentum tensor. That is to say that we are going to work with a particular case of the $f(R, T)$ gravity [19]. The $T$-dependence in a gravitational theory is related to the description of some quantum effects (conformal anomaly) 19, as we are going to revisit later on. It is also motivated by the possible existence of imperfect fluids in the universe. In fact, when putting $f(T)$ in the gravitational action, the field equations of the model can be cast in terms of an effective energy-momentum tensor whose extra terms are related to imperfections such as anisotropy, viscosity, elasticity etc.

The function $f(T)$ is, in principle, arbitrary, as the $f(R)$ function in the $f(R)$ gravity case. We are going to suppose here an exponential dependence for $T$ as $f(T) \sim e^{\chi T}$, with $\chi$ being a free parameter. Such an assumption is somehow motivated by exponential $f(R)$ gravity [20]. Anyhow, the assumption of dynamical fields exponentially entering the gravitational action is not an exclusivity of the exponential $f(R)$ gravity. In Ref. [21, a scalar field is playing the role of dark energy and is coupled to the Ricci scalar exponentially. In Ref. 22, it was proposed the substitution of $R$ by $\Lambda e^{\frac{R+L}{\Lambda}}$ in the gravitational action, with $L$ being the matter Lagrangian.

Our article is organized as follows: in Section II] we present the basic formalism of $R+e^{T}$ gravity and in Section III. we discuss the $R+e^{T}$ cosmology with a simple exact solution. The dynamical behaviour of the obtained model in the presented $R+e^{T}$ cosmology is further discussed in Section IV] In Section V, we confront the theoretical predictions of our model with observational Hubble dataset. In Section VI, we present the statefinder diagnostic of our model. Our final remarks and discussion are presented in Section VII. 


\section{II. $R+e^{T}$ GRAVITY}

The field equations of $e^{T}$ gravity will be obtained from the variation of the action

$$
S=\int d^{4} x \sqrt{-g}\left(\frac{R+\gamma e^{\chi T}}{16 \pi}+L\right),
$$

with $g$ being the metric determinant, $\gamma$ a constant and natural units are assumed. Such a variation with respect to the metric $g_{\mu \nu}$ yields

$$
G_{\mu \nu}=8 \pi T_{\mu \nu}^{\mathrm{eff}}
$$

with

$$
T_{\mu \nu}^{\mathrm{eff}}=T_{\mu \nu}+\frac{\gamma e^{\chi T}}{8 \pi}\left[\frac{g_{\mu \nu}}{2}+\chi\left(T_{\mu \nu}+p g_{\mu \nu}\right)\right],
$$

with $T_{\mu \nu}$ being the usual energy-momentum tensor of matter and $p$ the pressure.

The covariant derivative of the usual matter energymomentum tensor in (3) reads

$$
\begin{gathered}
\nabla^{\mu} T_{\mu \nu}= \\
-\frac{\gamma \chi e^{\chi T}}{8 \pi+\gamma \chi e^{\chi T}}\left[\chi\left(T_{\mu \nu}+p g_{\mu \nu}\right) \nabla^{\mu} T+\nabla^{\mu}\left(\frac{T}{2}+p\right) g_{\mu \nu}\right] .
\end{gathered}
$$

From (4), it can be seen that the energy-momentum tensor is not conserved in the present formalism. Such a feature can also be seen in other models [22]-25]. It has been shown that it can be related to a process of particles creation [26, 27].

Furthermore, from a completely different approach, in the cosmological models proposed in [28]-30], the mechanism behind the acceleration of the universe expansion is exactly the production of particles.

\section{III. $R+e^{T}$ COSMOLOGY}

With the set up of $R+e^{T}$ gravity discussed above, we now describe the $R+e^{T}$ cosmology for which, we consider the well-known Friedmann-Lemaître-RobertsonWalker metric 31-35 describing an isotropic and homogeneous universe. We make null the curvature of the spatial sector of the space-time, in accordance with observations of fluctuations of temperature in the cosmic microwave background radiation [4].

In parallel, we will consider the energy-momentum tensor of a perfect fluid. Also, in what concerns the material content of the universe in the present formalism, we desire to check if it is possible to describe the acceleration of the universe expansion as a consequence of the correction terms of the present gravitational model rather than as due to the presence of some exotic fluid such as dark energy permeating the universe. Therefore we should assume $p=0$ in the dynamical equations. This is equivalent to say that dust is presently the dominant component of the universe regulating the dynamics.

The above proceeding yields

$$
\begin{array}{r}
3 H^{2}=8 \pi \rho+\gamma e^{\chi \rho}\left(\frac{1}{2}+\chi \rho\right) \\
-2 \dot{H}=\left(8 \pi+\gamma \chi e^{\chi \rho}\right) \rho
\end{array}
$$

and

$$
\dot{\rho}\left[\frac{\gamma \chi e^{\chi \rho}}{8 \pi+\gamma \chi e^{\chi \rho}}\left(\frac{1}{2}+\chi \rho\right)+1\right]+3 H \rho=0 .
$$

In Eqs. (5)-(7) above, $H=\dot{a} / a$ is the Hubble parameter, $a$ is the scale factor, which dictates how distances evolve in the universe, and dots represent time derivatives. Moreover, it is important to notice that the usual Friedmann equations for pressureless matter (in the absence of the cosmological constant) are retrieved by taking $\gamma=0$.

From Equations (5) and (6) we get

$$
\begin{gathered}
\rho=\log \left(\frac{2 \mathcal{H}}{\gamma}\right)^{\frac{1}{\chi}}, \\
\dot{\rho}=\frac{2(\ddot{H}+3 H \dot{H})}{\chi \mathcal{H}},
\end{gathered}
$$

in which it was defined $\mathcal{H} \equiv 2 \dot{H}+3 H^{2}$.

Using Equations (8) and (9) into Equation (7) we obtain a dynamical equation in the form,

$$
\begin{array}{r}
2 \gamma(\ddot{H}+3 \dot{H} H)\left\{1+\frac{2 \chi \mathcal{H}^{2}\left[\frac{1}{2}+\log \left(\frac{2 \mathcal{H}}{\gamma}\right)\right]}{8 \pi+2 \chi \mathcal{H}}\right\} \\
+3 \log \left(2 \mathcal{H}^{2}\right) H=0 .
\end{array}
$$

A critical inspection of Equation 10 renders a simple solution for the condition

$$
\mathcal{H}>0
$$

which yields the scale factor $a(t)$ in the form of a hybrid expansion law (power and exponential laws) as,

$$
a(t)=\beta t^{\frac{2}{3}} e^{\alpha t}
$$

with $\alpha$ and $\beta$ being integrating constants.

The referred Hubble and deceleration parameters, with the latter defined as $q=-\ddot{a} a / \dot{a}^{2}$, such that negative values indicate an accelerated expansion, are

$$
\begin{gathered}
H(t)=\alpha+\frac{2}{3 t}, \\
q(t)=\frac{-3 \alpha t(4+3 \alpha t)+2}{(2+3 \alpha t)^{2}} .
\end{gathered}
$$

The cosmic dynamics of the obtained model can be discussed now. 


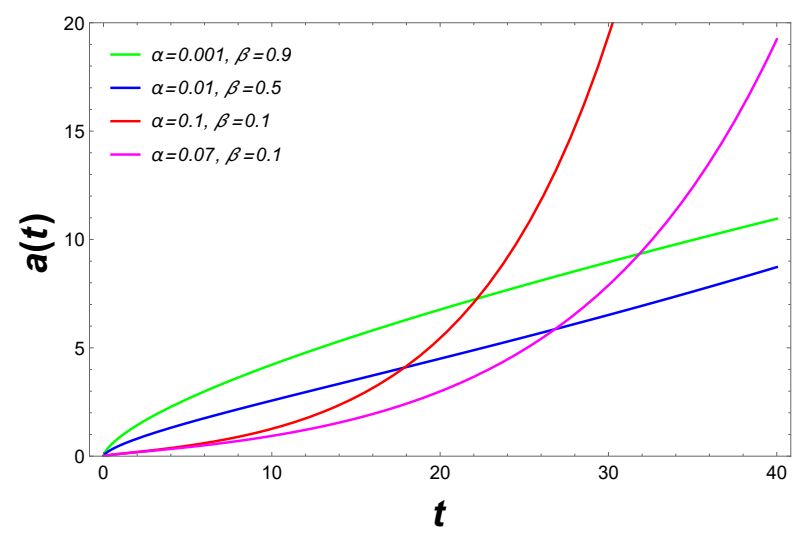

FIG. 1: Evolution of the scale factor $a(t)$ w.r.t cosmic time.

\section{COSMIC DYNAMICS}

With the exact solutions for the field equations in the $R+e^{T}$ cosmology, we shall now discuss the dynamics of the particular model obtained here. A quick analysis of the behavior of these geometrical parameters near the initial singularity and in the far future can be described in the following table

\begin{tabular}{|c|c|c|c|}
\hline Parameter & $a$ & $H$ & $q$ \\
\hline as $t \rightarrow 0$ & 0 & $\infty$ & $1 / 2$ \\
\hline as $t \rightarrow \infty$ & $\infty$ & $\alpha$ & -1 \\
\hline
\end{tabular}

TABLE I: Limiting values of geometrical parameters

From the above table I, we can observe that in this model the universe starts with a singularity and rapidly expands but with decreasing rate of expansion, which is suitable for structure formation. Also, it behaves like a de Sitter universe in the far future.

We can also plot these cosmological parameters w.r.t. cosmic time $t$ (in units of billions of years).

From the plot of the scale factor in Figure 1, we can see two different types of evolution with the choice of the model parameters $\alpha$ and $\beta$. The Hubble parameter in Figure 2 is almost the same independently of the $\alpha, \beta$ values and decreases rapidly. The rate of expansion can be seen in the plot of the deceleration parameter in Figure 3 for these choices of model parameters. For, the best fit values, in the following we shall constrain these model parameters with observations.

Further, the density parameter takes the form

$$
\rho=\log \left\{\left[\frac{2 \alpha(3 \alpha t+4)}{\gamma t}\right]^{1 / \chi}\right\}
$$

(recalling we are assuming that dust matter (with $p=0$ ) is filling the universe).

In order to confront our results with observations, it is convenient to rewrite all of these cosmological parameters in terms of the redshift $z$ rather than in terms of time. To do so, we use $a(t)=\frac{a_{0}}{1+z}$, with the present value of the scale factor $a_{0}=1$. The time-redshift relation for our obtained model can be expressed as

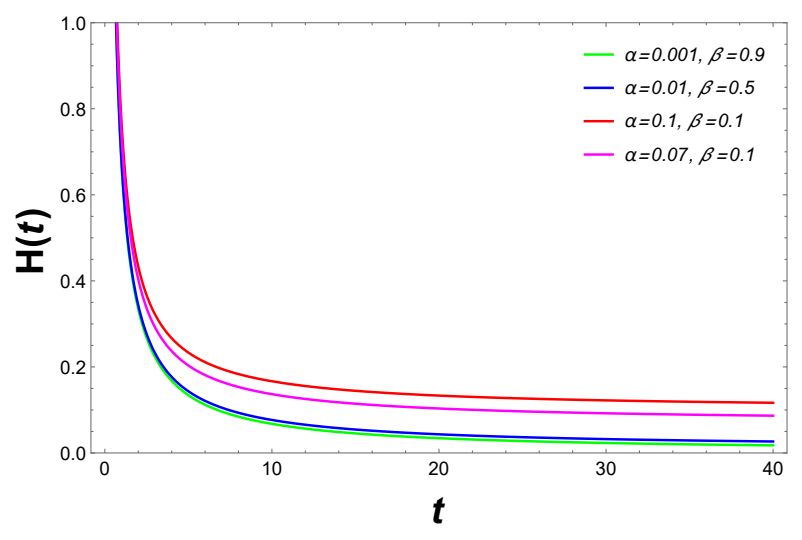

FIG. 2: Evolution of the Hubble parameter $H(t)$ w.r.t cosmic time.

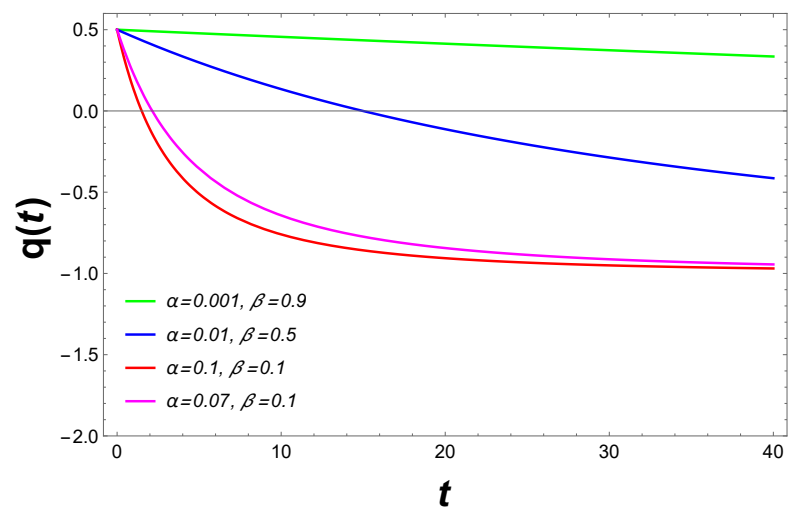

FIG. 3: Evolution of the deceleration parameter $q(t)$ w.r.t cosmic time.

$$
t=\frac{2}{3 \alpha} \mathcal{W}\left\{\frac{3 \alpha}{2} \frac{1}{[\beta(1+z)]^{3 / 2}}\right\},
$$

where $\mathcal{W}$ denotes the Lambert function, also known as "product logarithm". For the sake of simplicity, we will take, from now on, $\Gamma \equiv \frac{3 \alpha}{2} \frac{1}{[\beta(1+z)]^{3 / 2}}$.

Eqs. 13 and (14) now read

$$
\begin{gathered}
H(z)=\alpha\left[1+\frac{1}{\mathcal{W}(\Gamma)}\right], \\
q(z)=\frac{1}{2} \frac{1-2 \mathcal{W}(\Gamma)[2+\mathcal{W}(\Gamma)]}{[1+\mathcal{W}(\Gamma)]^{2}} .
\end{gathered}
$$

\section{CONFRONTATION WITH HUBBLE DATA}

In this section, we put constrains on the model parameters $\alpha$ and $\beta$ of the presented model in view of the observational Hubble dataset (OHD) $H(z)$.

We consider the OHD containing 57 data points of $H$ in the redshift range $0.07 \leqslant z \leqslant 2.36$ with the corresponding standard deviations $\sigma_{H}$. 31 points are obtained by the differential age techniques applied to evolving galaxies and 26 points are obtained by BAO and other methods 


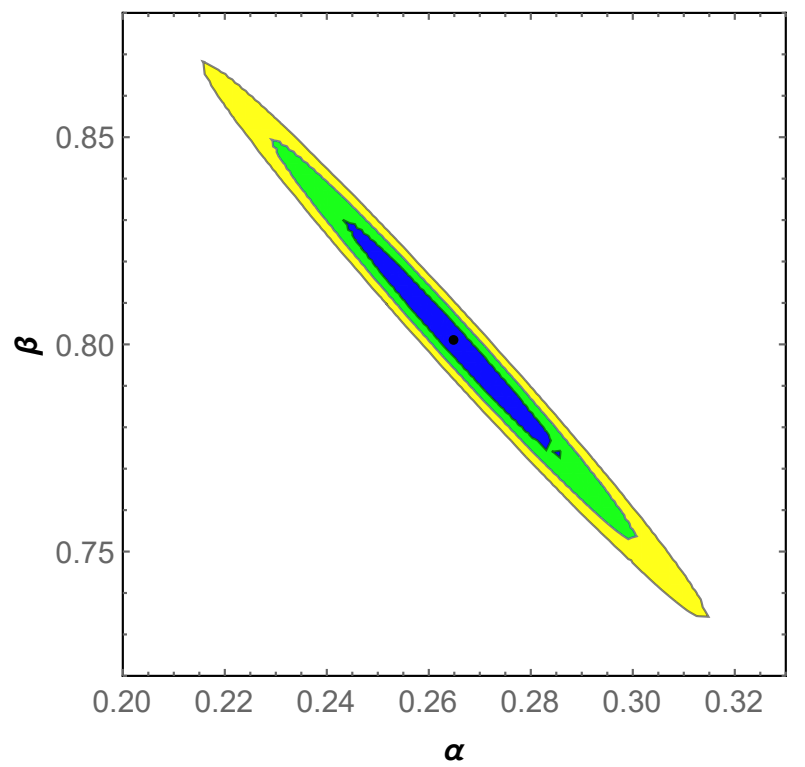

FIG. 4: Plots for $1 \sigma$ (blue shaded), $2 \sigma$ (green shaded) and $3 \sigma$ (yellow shaded) likelihood contours in the $\alpha$ - $\beta$ plane for the $R+e^{T}$ cosmological model.

[36. We take the present value of the Hubble parameter as $H_{0}=67.8 \mathrm{Km} / \mathrm{s} / \mathrm{Mpc}$, as suggested by Planck satellite results [37] to complete the dataset. In the calculation of $H(z)$ dataset, the mean values of the model parameters $\alpha$ and $\beta$ are determined by minimizing

$$
\chi_{O H D}^{2}\left(p_{s}\right)=\sum_{i=1}^{57} \frac{\left[H_{t h}\left(p_{s} ; z_{i}\right)-H_{o b s}\left(z_{i}\right)\right]^{2}}{\sigma_{H\left(z_{i}\right)}^{2}},
$$

where $p_{s}$ denotes the parameters of the model to be constrained, $H_{t h}$ denotes the theoretical (model based) values of the Hubble parameter, $H_{\mathrm{obs}}$ signifies the observed values of the Hubble parameter and $\sigma_{H\left(z_{i}\right)}$ is the standard error in each observed value. The summation runs over 57 observational data points at redshifts $z_{i}$ together with the $H_{0}$ value.

The likelihood contours in the $\alpha-\beta$ plane with $1 \sigma$, $2 \sigma$ and $3 \sigma$ errors are obtained for our model as shown in Fig,4. The best fit values of $\alpha$ and $\beta$ come out to be $\alpha=0.265005$ and $\beta=0.800925$, with minimum $\chi^{2}=$ 31.6247 .

The Hubble data with error bars can be seen in Fig 5 together with the curves of the $\Lambda \mathrm{CDM}$ and $R+e^{T}$ models. The figure clearly shows a nice fit to the OHD for the $R+e^{T}$ model with $\alpha=0.265005$ and $\beta=0.800925$.

One of the most important and crucial phenomena in the evolution of the Universe is the cosmological "phase transition" i.e. the time when the universe changes from a decelerating phase of expansion to an accelerating one. The model we obtained here indeed possesses the characteristic of cosmological phase transition for the constrained values of $\alpha$ and $\beta$ as one can see in Fig 6 .

From Fig 6 , one can observe that the phase transition from deceleration to acceleration occurs at $z_{t}=0.571166$. $z_{t}$ for our model and is in good agreement with the value predicted in [41-43]. Moreover, we can calculate the present value of the deceleration parameter as $q_{0}=-0.22$

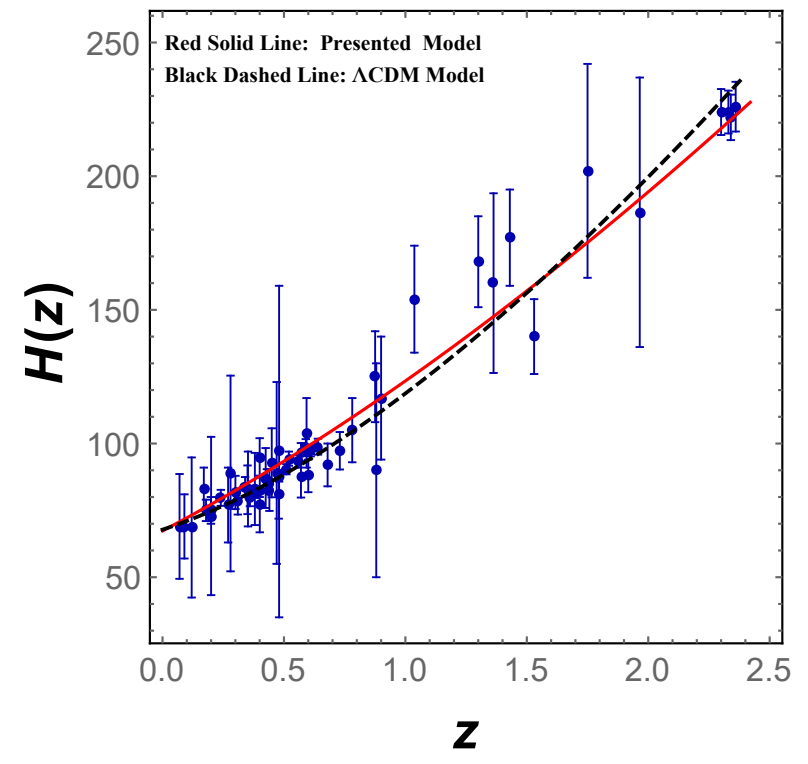

FIG. 5: 57 points of $H(z)$ dataset with error bars along with the present model (solid red line) and $\Lambda$ CDM model (black dashed line).

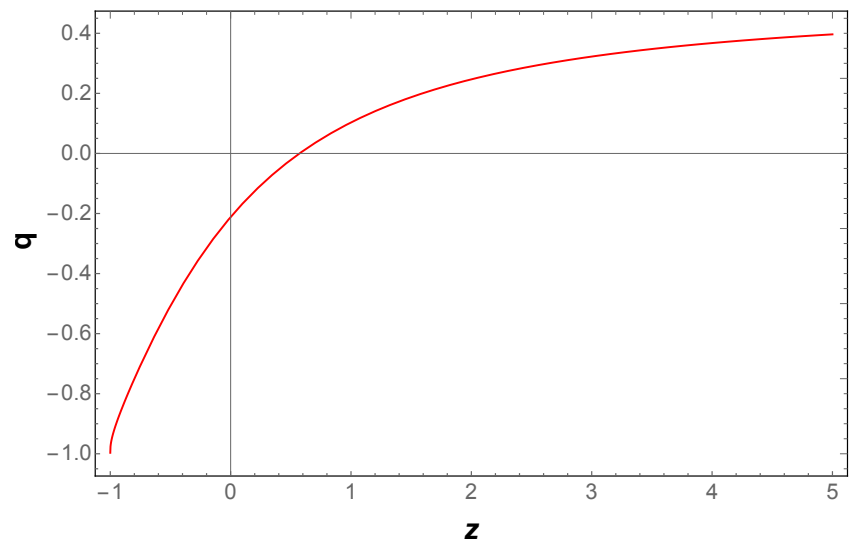

FIG. 6: Deceleration parameter vs. redshift, with $\alpha=$ 0.265005 and $\beta=0.800925$.

and is also in agreement with some recent constraints put on $q_{0}$ [44].

With the constrained values of the model parameters $\alpha$ and $\beta$, the plot of $\rho(z)$ is shown in Figure 7 .

\section{STATEFINDER DIAGNOSTIC}

The necessity of considering more general dark energy models than the standard one together with the increasing in the accuracy of cosmological observations has led V. Sahni and collaborators to introduce a cosmological diagnostic pair, namely statefinder pair $\{r, s\}[38,39$. This is constructed from the scale factor and its derivatives up to the third order and reads

$$
r=\frac{\dddot{a}}{a H^{3}},
$$




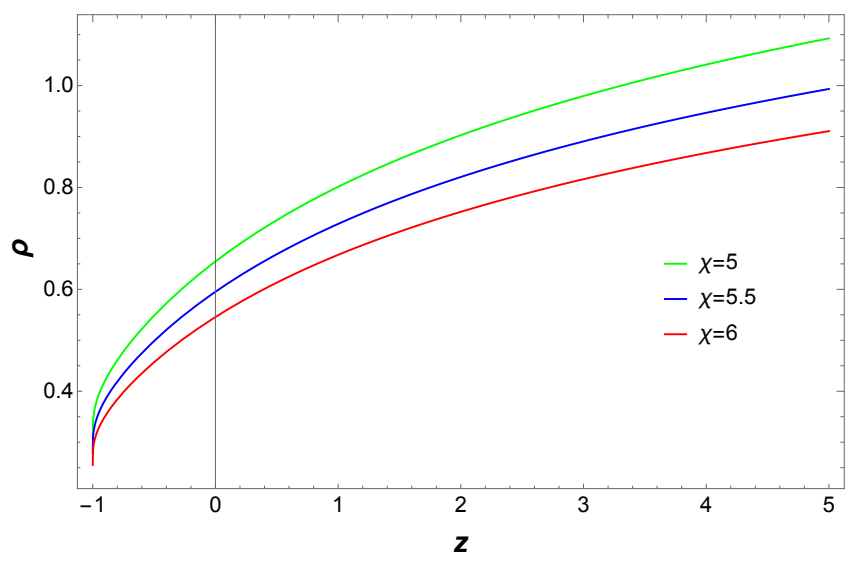

FIG. 7: Density vs. redshift, with $\alpha=0.265005$ and $\beta=$ 0.800925 .

$$
s=\frac{1}{3}\left(\frac{-1+r}{-\frac{1}{2}+q}\right),
$$

where $q \neq \frac{1}{2}$.

An useful way of distinguishing different cosmological models that have similar kinematics is to plot the evolution trajectories of the $q-r$ and $r-s$ pairs.

For our model, the pair $r, s$ reads

$$
\begin{gathered}
r=2 \frac{4+9 \alpha t[-1+\alpha t(2+\alpha t)]}{(2+3 \alpha t)^{3}}, \\
s=\frac{4}{8+9 \alpha t(2+\alpha t)} .
\end{gathered}
$$

We observe the trajectories of the hybrid scale factor obtained in our model in Figs 8,9, in which the values of $\alpha$ and $\beta$ were chosen in accordance to the previous section results. They follow a pattern similar to that of power law cosmology. The trajectories in $s-r$ plane in the power law cosmology (see Ref. 40]) start from SCDM (standard cold dark matter $(\Lambda=0)$ ) point and follows up the $\Lambda$ CDM model while this hybrid model is deviated from the SCDM and follows up the $\Lambda$ CDM model. Similarly, the trajectories in the $q-r$ plane can be seen and compared with the power law cosmology.

\section{DISCUSSION}

The longstanding cosmological constant problem remains as one of the greatest observational issues of Physics. An interesting form to alleviate it is by considering the cosmological "constant" as time varying (for instance, Ref. 45]). Besides changing the gravitational aspects of gravity, as deeply mentioned in Section [1] one can also infer the cosmic acceleration by assuming the Universe is filled with some fluid that follows the Chaplygin gas equation of state [46]-50]. This is also an alternative to describe cosmic acceleration with no cosmological constant, and in fact it can provide an unified alternative to dark energy and dark matter.

Here we have addressed the cosmic acceleration through an extended theory of gravity named $f(R, T)$

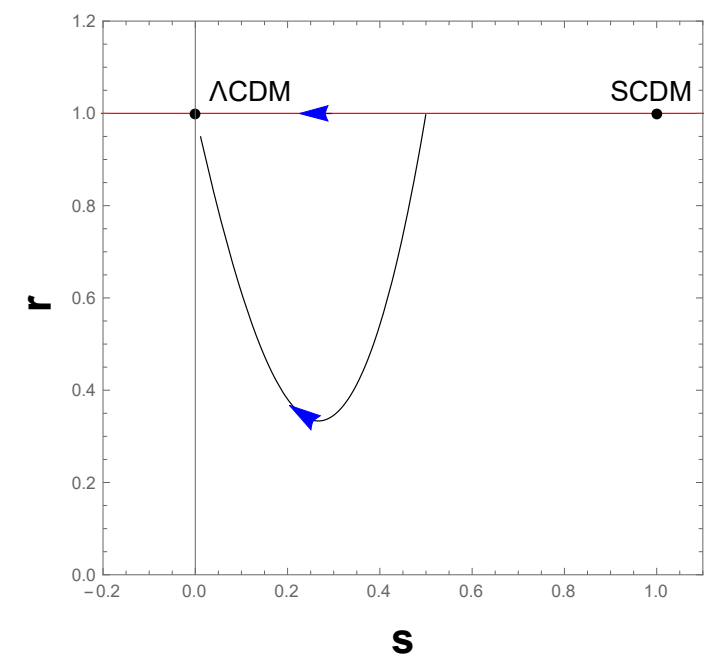

FIG. 8: Variation of $s$ vs. $r$ for the $R+e^{T}$ cosmological model with $\alpha=0.265005$ and $\beta=0.800925$.

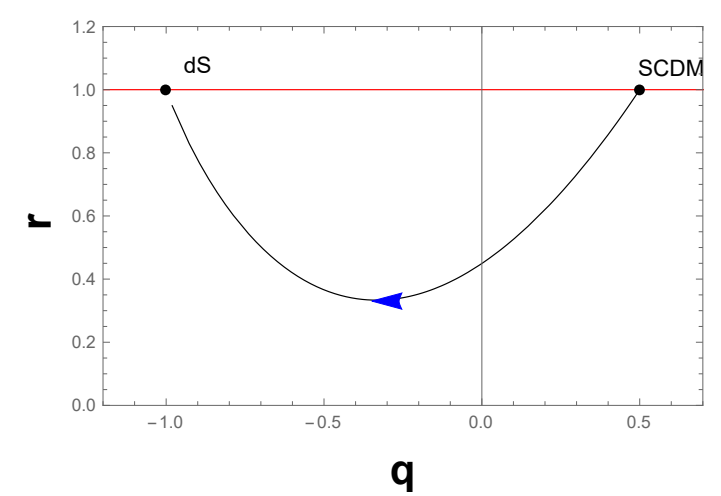

FIG. 9: Variation of $q$ vs. $r$ for the $R+e^{T}$ cosmological model with $\alpha=0.265005$ and $\beta=0.800925$. "dS" stands for de Sitter universe.

gravity that allows the inception of material terms dependent on $T$ in the gravitational action. As a novelty in the literature, we considered an exponential dependence for the trace of the energy-momentum tensor.

We have used here the Hubble parameter data obtained by different age techniques applied to evolving galaxies and constrained the model parameters $\alpha$ and $\beta$ in our model respectively as $\alpha=0.265005$ and $\beta=$ 0.800925 , with minimum $\chi^{2}=31.6247$ based on Bayesian statistics. Moreover, we have obtained the likelihood contours at $1 \sigma, 2 \sigma$ and $3 \sigma$ confidence levels using the $\mathrm{OHD}$ as shown in the $\alpha-\beta$ plane of Figure 4 . We have compared our model with the $\Lambda$ CDM model. We found that our model is fitting well the $\mathrm{OHD}$ with the constrained values of $\alpha$ and $\beta$ and does not deviate significantly from $\Lambda \mathrm{CDM}$ (see Figure 5), though for higher values of redshift the present model clearly presents a better adjust with observations.

The statefinder diagnostic, which was here obtained for the $R+e^{T}$ cosmology, is generally used to compare various dark energy models and their deviations from $\Lambda$ CDM and SCDM models. We have shown the time and redshift evolutions of various cosmological parameters. We have demonstrated the phase transition redshift through 
graphical representation (Fig 6). All of these features elevate the $R+e^{T}$ cosmology to the level of an important alternative to describe the cosmic acceleration with no need of a cosmological constant.

Finally, by power expanding $e^{T}$ in the model action, one obtains $R+T+1+\Theta\left(T^{2}\right)$. In the first order approximation and properly inserting the right units, the third term in the expansion reads as a cosmological constant, which naturally appears in the model. Since the $f(R, T)=R+T$ cosmology has been discarded from observational data [51, this expansion appears as a good alternative to revive this scenario.

\section{Acknowledgements}

PHRSM would like to thank CAPES for financial support. PKS acknowledges CSIR, New Delhi, India for financial support to carry out the Research project [No.03(1454)/19/EMR-II Dt.02/08/2019].
[1] E. Hubble, Proc. Nat. Acad. Sci. U.S.A. 15 (1929) 168173.

[2] A.G. Riess et al., Astron. J. 116 (1998) 1009-1038.

[3] S. Perlmutter et al., Astrophys. J. 517 (1999) 565-586.

[4] Planck Collaboration: N. Aghanim et al., Astron. Astrophys. 607 (2017) A95.

[5] S. Weinberg, Rev. Mod. Phys. 61 (1989) 1.

[6] P.J. Peebles, B. Ratra, Rev. Mod. Phys. 75 (2003) 559.

[7] T. Padmanabhan, Phys. Rep. 380 (2003) 235-320.

[8] V.A. Bednyakov, Is it possible to discover a dark matter particle with an accelerator?, Phys. Part. Nucl. 47 (2016) 711-774. https://doi.org/10.1134/S1063779616050026

[9] Y. Mambrini, E. Nezri, Eur. Phys. J. C 50 (2007) 949968.

[10] A. Einstein, Sitzungsber. Preuss. Akad. Wiss. Berlin(1915) 844-847.

[11] A. De Felice, S. Tsujikawa, Liv. Rev. Rel. 13 (2010) 3.

[12] S. Capozziello, M. de Laurentis, Phys. Rep. 509 (2011) 167-321.

[13] S. Nojiri et al., Phys. Rev. D 74 (2006) 046004.

[14] Y. Shirasaki et al., Publ. Astron. Soc. Jap. 68 (2016) 23.

[15] S. Capozziello et al., J. Cosm. Astrop. Phys. 2013 (2013) 024.

[16] D. C. Rodrigues et al., Month. Not. Roy. Astron. Soc. 445 (2014) 3823-3838.

[17] T. Chiba, Phys. Lett. B 575 (2003) 1-3.

[18] S. Nojiri, S.D. Odintsov, Phys. Lett. B 659 (2008) 821826.

[19] T. Harko et al., Phys. Rev. D 84 (2011) 024020.

[20] S.D. Odintsov et al., Eur. Phys. J. C 77 (2017) 862.

[21] V. Pettorino et al., J. Cosm. Astrop. Phys. 2005 (2005) 014.

[22] T. Harko, F.S.N. Lobo, Eur. Phys. J. C 70 (2010) 373.

[23] A. M. Oliveira et al., Phys. Rev. D 92 (2015) 044020.

[24] T. Harko et al., Mod. Phys. Lett. A 26 (2011) 1467.

[25] R. Mignani et al., Gen. Rel. Grav. 29 (1997) 1049-1073.

[26] T. Harko et al., Eur. Phys. J. C 75 (2015) 386.

[27] T. Harko, Phys. Rev. D 90 (2014) 044067.
[28] G. Steigman et al., An accelerating cosmology without dark energy, J. Cosm. Astrop. Phys. 2009 (2009) 033. https://doi.org/10.1088/1475-7516/2009/06/033

[29] J.A.S. Lima et al., J. Cosm. Astrop. Phys. 1011 (2010) 027.

[30] J.A.S. Lima et al., Phys. Rev. D 86 (2012) 103534.

[31] A. Friedmann, Phys. A 10 (1922) 377â€"386.

[32] A. Friedmann, Zeits. Phys. A 21 (1924) 326â€"332.

[33] G. Lemaître, Annal. Soc. Scient. Brux. A47 (1927) 4959.

[34] H.P. Robertson, Astrophys. J. 82 (1935) 284.

[35] A.G. Walker, Proc. Lon. Math. Soc. 42 (1937) 90-127.

[36] G. S. Sharov, V. O. Vasiliev, Mathematical Modelling and Geometry 6(1) (2018) 1-20.

[37] Planck Collaboration, Astron. \& Astrophys. 594 (2016) A13.

[38] V. Sahni et al., JETP Lett. 77 (2003) 201-206.

[39] U. Alam et al., Mon. Not. R. Astron. Soc. 344 (2003) 10571074.

[40] Sarita Rani et al., J. Cosmol. Astropart. Phys. 2015 (2015) 031.

[41] S. Capozziello, O. Farooq, O. Luongo, B. Ratra, Phys. Rev. D, 90 (2014) 044016.

[42] S. Capozziello, O. Luongo, E. N. Saridakis B., Phys. Rev. D, 91 (2015) 124037.

[43] O. Farooq, F. Madiyar, S. Crandall, B. Ratra, Astrophys. J., 835 (2017) 26.

[44] R. Giostri et al., J. Cosm. Astrop. Phys. 03 (2012) 027.

[45] S. Basilakos et al., Phys. Rev. D, 80 (2009) 083511.

[46] M.C. Bento et al., Phys. Rev. D, 66 (2002) 043507.

[47] V. Gorini et al., Phys. Rev. D, 67 (2003) 063509.

[48] U. Debnath et al., Class. Quant. Grav. 21 (2004) 56095618.

[49] M.C. Bento et al., Phys. Rev. D, 67 (2003) 063003.

[50] M.C. Bento et al., Phys. Lett. B 575 (2003) 172-180.

[51] H. Velten and T.R.P. Caramês, Phys. Rev. D, 95 (2017) 123536. 